\title{
L'éboulement du mont Granier en novembre 1248
}

M. PANET

43 bis, av. Le Nôtre 92330 Sceaux marc.panet@gmail.com
Le grand éboulement qui s'est produit au mont

Granier dans la nuit du 24 au 25 novembre 1248 demeure un sujet d'étude pour les géomécaniciens. De nombreuses observations géologiques, morphologiques et hydrogéologiques ont été faites sur le site de cette grande catastrophe naturelle. Plusieurs mécanismes peuvent être envisagés pour expliquer cet éboulement. L'hypothèse la plus communément admise est celle d'un grand glissement dans les marnes qui aurait entraîné la rupture de la falaise calcaire. Mais on peut également envisager d'énormes coulées de boue générées par l'impact de l'éboulement de la falaise urgonienne.

Mots-clés : éboulement, glissement, coulée de boue falaise.

\section{The mount Granier rockslide, November 1248}

The large rock fall of $\alpha$ mont Granier $»$ which occurred in the night 24-25 November 1248 is still a matter of discussion in geomechanics. A large number of geological, geomorphological and hydrogeological observations have been carried out on the site of this huge natural catastrophe. Several mechanisms may be proposed to explain the rock fall. The most common assumption is a large slide in the underlying marl formation bringing about the fall of the limestone cliff. However, the development of very large debris flows due to the impact of the rock fall may be considered.

Key words: rockfall, slide, mudflow, cliff. 
Le mont Granier qui culmine à $1933 \mathrm{~m}$ limite, au nord-est, le massif de la Chartreuse. Son imposante face nord qui domine la vallée du Grésivaudan, la combe de Savoie et la cluse de Chambery résultent d'un gigantesque éboulement (Fig. 1).

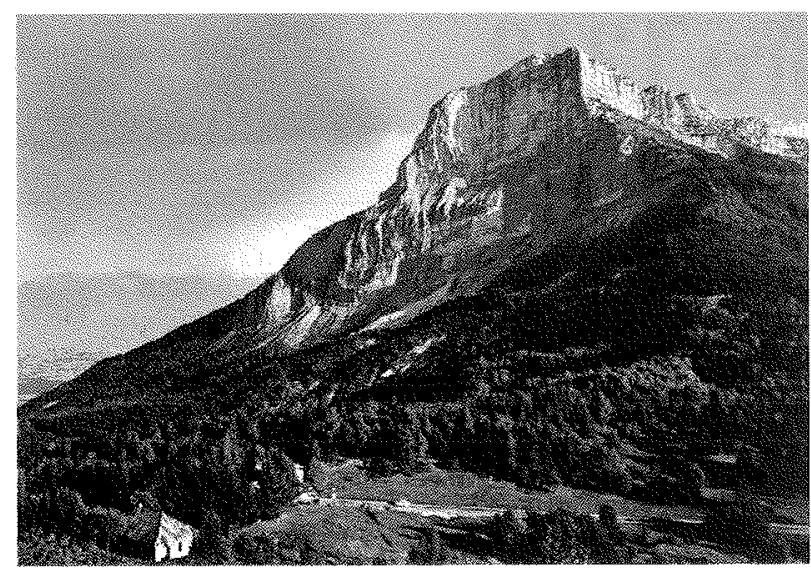

fra. 1 Vue de la face nord du mont Granier.

\section{Introduction}

Dans la nuit du 24 au 25 novembre 1248, la limite septentrionale du mont Granier s'est écroulée, créant une falaise haute de près de $700 \mathrm{~m}$ et large de $600 \mathrm{~m}$ à $700 \mathrm{~m}$. Cette catastrophe provoqua la destruction de cinq villages du décanat de Savoie; selon les estimations les plus récentes, le nombre de victimes aurait été supérieur à un millier. Plusieurs thèses ont été avancées pour expliquer cet éboulement. La thèse proposée par Jean Goguel et Albert Pachoud (1972) est la plus étayée et la plus communément admise (Cruden et Antoine, 1984 ; Goguel et Pachoud, 1972 ; Panet, 2000).

\section{2}

\section{Les données stratigraphiques}

Les formations calcaires allant du Barrémien au Valanginien affleurent sur la falaise septentrionale du mont Granier (carte géologique à 1/50 000 de Montmélian), soit de haut en bas :

- les calcaires de l'Urgonien supérieur qui ne sont représentés qu'en faible épaisseur au sommet de la falaise $\left(\mathrm{n}_{5} \mathrm{U}\right)$;

- les calcaires roux à orbitolines du Bédoulien épais de 5 à $20 \mathrm{~m}\left(\mathrm{n}_{5} 0\right)$;

- les calcaires cristallins blancs ou rosés de l’Urgonien inférieur épais de $200 \mathrm{~m}\left(\mathrm{n}_{4} \mathrm{U}\right)$;

- les calcaires roux et bien lités de la base de l'Urgonien de 40 à $100 \mathrm{~m}$ d'épaisseur $\left(\mathrm{n}_{4} \mathrm{a}\right)$;

- les marnes à Spatangues de l'Hauterivien épaisses de 70 à $100 \mathrm{~m}\left(\mathrm{n}_{3}\right)$;

- les calcaires à silex du Valanginien supérieur de $40 \mathrm{~m}$ d'épaisseur $\left(\mathrm{n}_{2} \mathrm{~S}\right)$;

- les calcaires bicolores du Fontanil épais de 150 m $\left(n_{2} F^{-1}\right)$.

La base de la falaise est constituée par la puissante couche des marnes de Narbonne épaisse de $500 \mathrm{~m}$ environ $\left(\mathrm{n}_{1-2} \mathrm{M}\right)$.

\section{3}

\section{Les données structurales}

La bordure est du massif de la Chartreuse correspond à une structure synclinale perchée d'axe sensiblement nord-sud qui plonge de l'ordre de $10^{\circ}$ vers le nord à laquelle succècle une structure anticlinale dont l'axe passe par le col du Granier (Fig. 2). Dans son extrémité vers la zone de l'éboulement, les couches ont un pendage uniforme de $10^{\circ}$ à $15^{\circ}$ vers le nord-est.

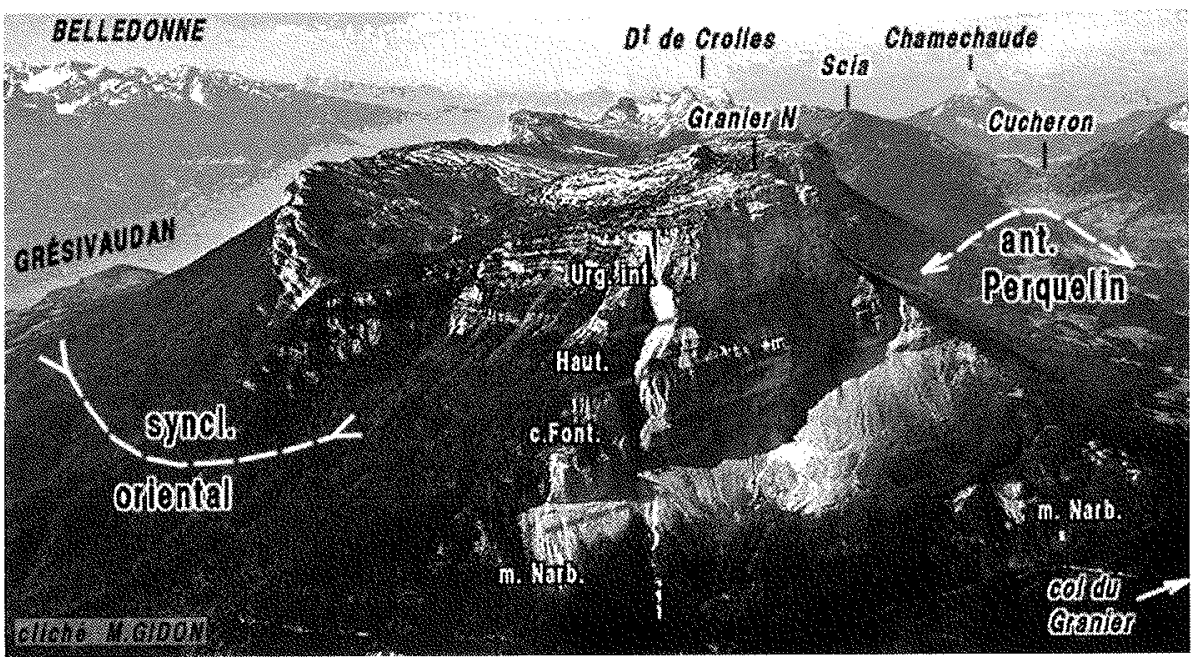

fic 2. Géologie de la terminaison septentrionale de la Grande Chartreuse (Gidon). 
La structure synclinale est affectée par un important réseau de décrochements dextres NE-SW, notamment les accidents du col de l'Alpette et de Pin Chérin. Un de ces décrochements pourrait correspondre à la falaise du mont Granier. Le rejet latéral de ces décrochements est de l'ordre du kilomètre. La composante verticale de ces failles abaisse le compartiment sud.

Le massif est également affecté par un réseau de diaclases subverticales extrêmement denses de direction $\mathrm{N} 150$ et $\mathrm{N} 80$.

\section{Les données hydrogéologiques}

Les données stratigraphiques faisant alterner des formations calcaires et des formations marneuses imperméables conduisent à considérer l'existence de deux aquifères :

- un aquifère supérieur dans les calcaires de l'Urgonien ayant pour mur les marnes à Spatangues de l'Hauterivien. Les dolines et avens sur le plateau, les chenaux visibles sur la falaise montrent le caractère karstique de l’Urgonien ;

- un aquifère inférieur dans les calcaires du Fontanil ayant pour mur les marnes valanginiennes. A cet aquifère correspondent de nombreuses sources sur le versant.

\section{5}

\section{La morphologie du versant}

Les parties supérieure et inférieure de la falaise qui correspondent aux formations calcaires de l'Urgonien et au calcaire du Fontanil forment des barres subverticales. Entre les deux barres, les formations marneuses de l'Hauterivien présentent une forte pente.

Le pied de la falaise est tapissé d'éboulis rocheux récents correspondant à de petits éboulements et chutes de blocs de la falaise. Un éboulement plus important dont la falaise garde la cicatrice se produisit en 1953. Selon J. Goguel et A. Pachoud, les blocs de cet éboulement ne dépassèrent pas une distance de $500 \mathrm{~m}$ à vol d'oiseau.
L'épanchement de l'écroulement de 1248 est beaucoup plus considérable puisque des blocs rocheux se retrouvent jusqu'à une distance de $7,3 \mathrm{~km}$ à vol d'oiseau dans la zone dite (c des abîmes de Myans ».

Les levers de terrain de J. Goguel et A. Pachoud (1972) montrent que les blocs rocheux de la partie inférieure de la falaise (blocs néocomiens) se sont principalement épanchés sur une distance qui n'excède pas $4 \mathrm{~km}$ alors que des blocs urgoniens de la partie supérieure ont parcouru des distances supérieures à $7 \mathrm{~km}$ (Fig. 3).

Les blocs rocheux sont noyés dans une gangue de terrains marneux. Ils donnent à toute la zone de l'épanchement un aspect chaotique. De très gros blocs urgoniens émergent dans la zone des « abîmes de SaintAndré ») et forment de nombreux « molards ».

Le CETE de Lyon (2004) a mis en évidence par photo-interprétation un autre trait morphologique important : la présence au pied de la falaise d'une vaste dépression avec des contre-pentes vers le sud. Cette dépression pourrait être la cicatrice de l'impact de la chute de l'énorme pan de falaise.

\section{6}

\section{Les mécanismes possibles de l'éboulement} ment :

L'analyse de cet éboulement comporte nécessaire- l'étude de la rupture de la falaise ;

- le comportement des marnes valanginiennes sousjacentes.

\section{s.ti}

\section{La rupture de la falaise}

La morphologie du versant avant l'éboulement est évidemment très difficile à déterminer ; on peut toutefois faire référence à la tentative faite par J. Goguel et A. Pachoud (1972). On peut donc admettre que le pied de la rupture de la falaise se situe vraisemblablement entre $1200 \mathrm{~m}$ et $1300 \mathrm{~m}$ alors que la cote du som-

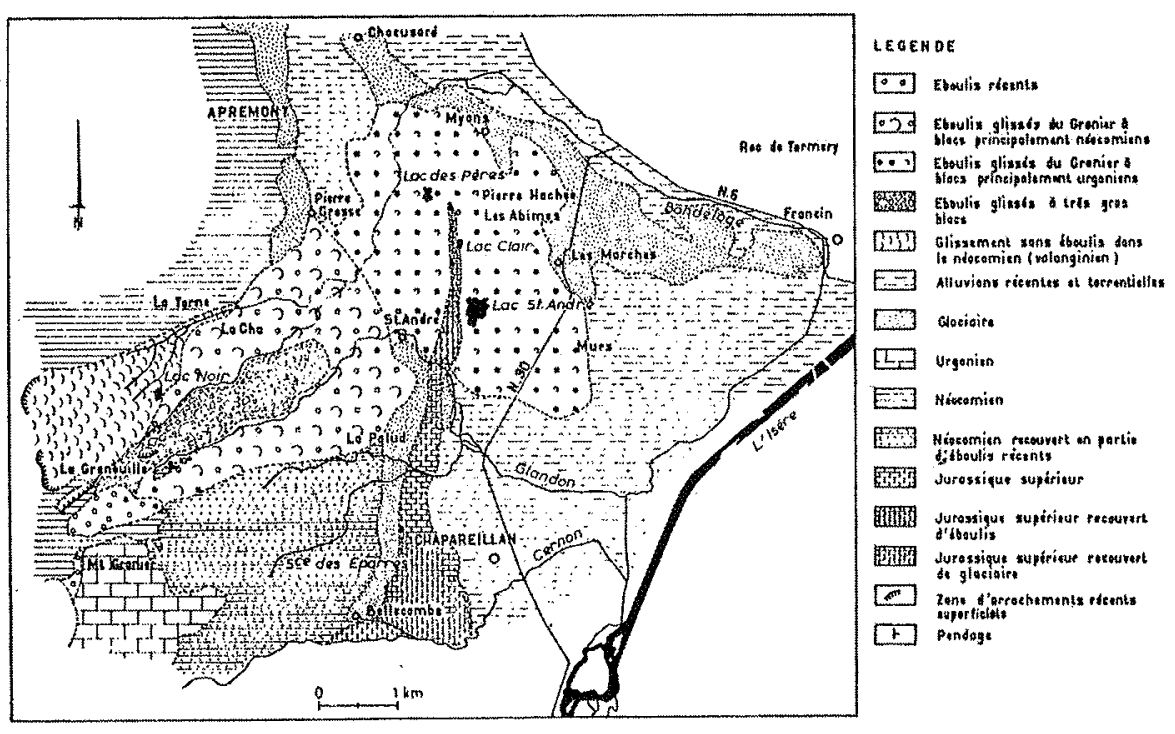

f6. 3 Carte de l'éboulement du mont Granier (J. Goguel, A. Pachoud, 1972). 
met varie entre $1900 \mathrm{~m}$ et $1850 \mathrm{~m}$. La hauteur de la falaise qui s'est écroulée peut donc être estimée entre $700 \mathrm{~m}$ et $550 \mathrm{~m}$. La largeur de la cicatrice varie entre $500 \mathrm{~m}$ au sommet et $750 \mathrm{~m}$ au pied. L'épaisseur de cette masse est la plus difficile à évaluer ; $25 \mathrm{~m}$ d'épaisseur moyenne doit constituer une borne inférieure, mais on ne peut pas exclure une valeur de $50 \mathrm{~m}$. Sur ces bases, le volume de la masse de la falaise qui s'est éboulée varie entre $10.10^{6} \mathrm{~m}^{3}$ et $20.10^{6} \mathrm{~m}^{3}$. On peut retenir en première approximation $15.10^{6} \mathrm{~m}^{3}$. Cette valeur raisonnable est toutefois assez largement supérieure aux estimations antérieures (5.10 $\mathrm{m}^{3}$ ) (Gruden et Antoine, 1984; Goguel et Pachoud, 1972).

En se référant à la classification proposée L. Rochet (1987) (Fig. 4) fondée sur le volume des masses en mouvement, un éboulement de cette ampleur se classe dans les éboulements en très grande masse pour lesquels, au cours de la propagation, les interactions entre les éléments rocheux deviennent prépondérantes; la dissipation de l'énergie au sein de l'éboulement décroit avec le volume, se traduisant par un phénomène de fluidisation. Les blocs rocheux peuvent alors franchir des distances considérables.

La distance maximale que peuvent atteindre les blocs peut être caractérisée par le rapport entre, d'une part, la différence de cote entre le sommet de la rupture et les blocs ayant franchi la plus grande distance et, d'autre part, la longueur de la projection horizontale de cette distance (en allemand, le Fahrboschung, selon Heim (1932)). Ce coefficient F (Fig. 5) décroît avec le volume de l'instabilité. Dans le cas de l'écroulement du Granier, ce coefficient peut être estimé à 0,22 . Selon le graphique établi par Scheidegger (1973) sur des bases statistiques, cette valeur est un peu faible située

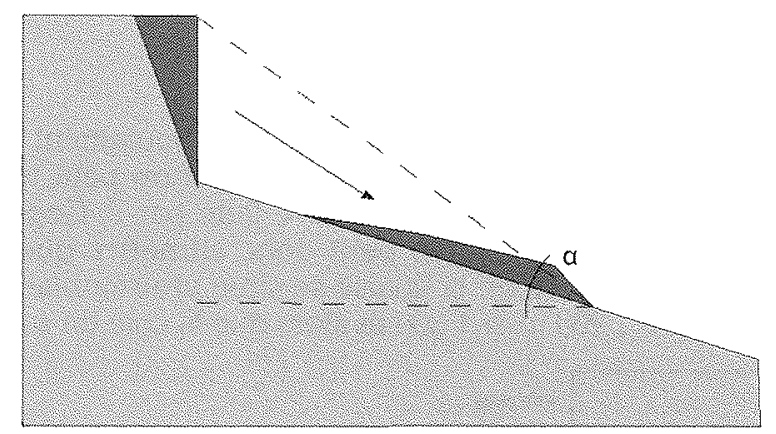

Ho. 5 Coefficient $F=\operatorname{tg} \alpha$ de la distance de parcours des éléments rocheux. à la limite inférieure du fuseau. Il convient en outre de remarquer que la propagation de l'éboulement ne s'est pas faite vers le NW dans une direction perpendiculaire à la falaise mais vers l'ENE. La seule propagation de l'éboulement de la falaise explique difficilement la distance franchie par les blocs urgoniens.

Dans le mécanisme de rupture de la falaise, il conviendrait de distinguer:

- la rupture de la falaise urgonienne suivant vraisemblablement un décrochement $\mathrm{N} 60^{\circ}$;

- la rupture par cisaillement des marnes hauteriviennes, le plan de rupture ayant un pendage de l'ordre de $40^{\circ}$;

- la rupture des calcaires du Fontanil suivant également un décrochement $\mathrm{N} 60^{\circ}$.

L'analyse de ces ruptures est difficile en l'absence de données sur la répartition des pressions interstitielles. Dans la falaise urgonienne, le drainage se fait naturellement par l'important réseau karstique, mais on ne peut pas exclure des mises en charge locales. Par contre une mise en charge des discontinuités dans le calcaire du Fontanil est probable. La mise en charge du calcaire du Fontanil épais de $150 \mathrm{~m}$ aurait pu provoquer le glissement des marnes valanginiennes qui aurait alors provoqué la rupture de la falaise. Le fait générateur serait alors le glissement dans les marnes valanginiennes.

L'analyse de la succession de ces ruptures ou de leur simultanéité reste à faire. Si la rupture de la partie supérieure de la falaise s'était produite avant la rupture de la partie inférieure on devrait trouver des blocs urgoniens en dessous de blocs de calcaire du Fontanil. Une telle observation n'a pas été mentionnée.

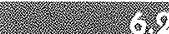

\section{Le comportement des marnes valanginiennes}

L'hypothèse, faite par J. Goguel et A. Pachoud (1972), d'un grand glissement sur un plan de stratification dans les marnes valanginiennes est la plus retenue. Le pendage du plan de stratification est toutefois faible de l'ordre de $12^{\circ}$.

Le mécanisme proposé par P. Habib (1967) pour expliquer les vitesses considérables atteintes par le glissement du mont Toc dans la retenue du barrage du Vaïont a été repris par J. Goguel (1969). L'impact de l'éboulement de la falaise a créé un déplacement

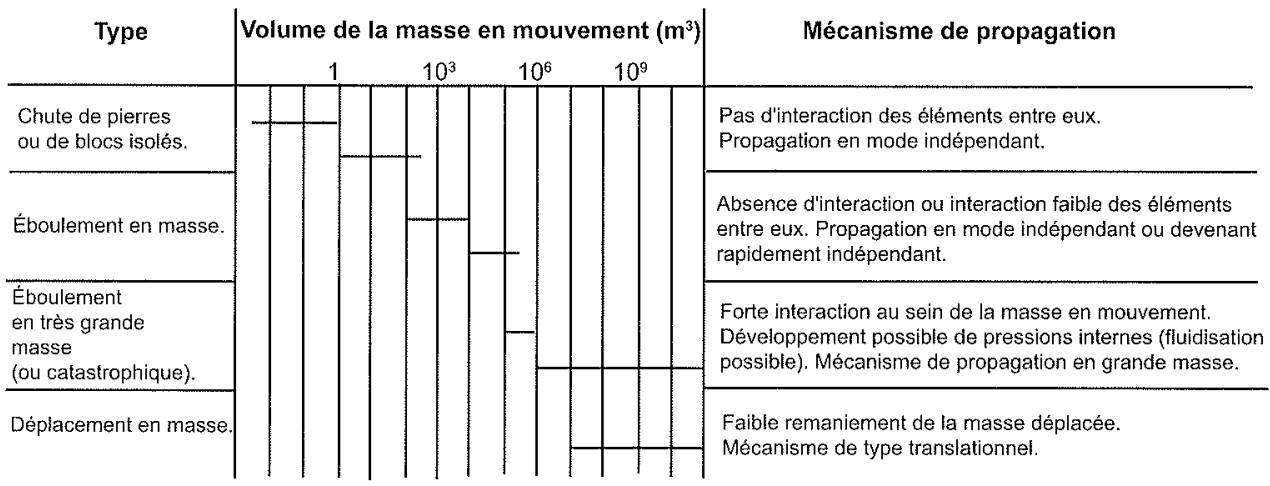


suffisant pour provoquer la vaporisation de l'eau interstitielle dans les marnes, le glissement se développant alors sur un coussin de vapeur. Le fait générateur serait alors l'éboulement de la falaise.

Enfin on ne peut pas exclure une troisième hypothèse. L'énergie potentielle d'une masse de $15.10^{6} \mathrm{~m}^{3}$ tombant en moyenne de $300 \mathrm{~m}$ de haut est considérable. Une partie de cette énergie se dissipe dans le mécanisme de rupture et clans les interactions entre blocs dans leur trajectoire aérienne. Cependant l'énergie disponible à l'impact demeure énorme. La chute de la falaise a pu provoquer un énorme cratère dans les colluvions et les marnes valanginiennes. Les chroniques de l'époque rapportent des précipitations excep- tionnelles dans les jours précédents et pendant la catastrophe. Le remaniement d'un volume important de terrains dû à l'impact de l'éboulement peut alors être à l'origine d'énormes coulées de boues entraînant sur une certaine distance les blocs de la falaise.

Les données disponibles me paraissent insuffisantes pour trancher définitivement en faveur de l'une ou de l'autre de ces hypothèses ou de leur combinaison. Des recherches complémentaires sur les conditions de rupture de la falaise et la structure et l'état des éboulis dans la zone d'épanchement de l'éboulement pourraient permettre de mieux comprendre les mécanismes de cet éboulement exceptionnel dont les mécanismes sont loin d'être complètement élucidés.

\section{Bibliographie}

CETE Lyon - L'écroulement du mont Granier (Savoie). Inventaire national des glissements anciens par photo-interprétation 2004.

Gruden D.M., Antoine P. - The Slide from Mont Granier, Isère and Savoie, France on November 24, 1248. Vol. 1, Toronto, 1984, p. $475-481$

Goguel J. - Le rôle de l'eau et de la chaleur dans les phénomènes tectoniques. Rev. Géogr. Phys. Géol. Dyn., Fr. (2), 11, n², 1969 , p. 153-163

Goguel J., Pachoud A. - Géologie et dynamique de l'écroulement du mont Granier dans le massif de Chartreuse en novem- bre 1948. Bulletin du BRGM, 2e série, Section III, $n^{\circ} 1,1972$, p. 29-38.

Habib P. - Sur un mode de glissement des massifs rocheux. C. R. Acad. Sci. Paris, 264, 1967, p. 151-153.

Panet M. - Les risques d'instabilité de masses rocheuses sur les versants des Alpes. Actes du colloque La Sécurité des Ouvrages, Hommage à Pierre Londe. Presses des Ponts, 2000.

Rochet L. - Application des modèles numériques de propagation à l'étude des éboulements rocheux. Bull. Liaison Laboratoire des Ponts et Chaussées, $n^{\circ} 150-151,1987$, p. 84-95.
Rochet Bouzid I. - Contribution à l'étude des écoulements granulaires appliqués aux éboulements rocheux en grande masse. Thèse de doctorat de l'université Claude-Bernard, Lyon, soutenue le 9 décembre 1999 . $\mathrm{N}^{\circ}$ d'ordre : 336-99.

Scheidegger A.E. - On the prediction of the reach and velocity of catastrophic landslides. Rock Mechanics, 5, 1973, p. 231-236.

L'éboulement du Granier et le sanctuaire de Myans - Actes du colloque de Myans, 5,6 et 7 juin 1998 . 